\title{
A method to measure enforcement effort in shipping with incomplete information
}

\author{
Xichen Ji¹, Jan Brinkhuis², Sabine Knapp ${ }^{3}$
}

Econometric Institute Report 2014-12 revised

Date: $8^{\text {th }}$ December 2014

\begin{abstract}
Current methods in the shipping industry to evaluate performance do not account for differences in fleet profiles of registries such as age, size or ship type and not for bad luck. This can lead to unfair evaluation of enforcement efforts of the international standards. Furthermore, incentives to improve performance are concentrated on decreasing detentions rather than incidents. This article proposes a new method to a longstanding problem to evaluate performance that rectifies shortcomings of the method currently used. The proposed method measures the enforcement effort by means of proxy variables and introduces incentives for improvement that go beyond the currently used 'detention'. The aim is to provide a fair and transparent way. The proposed method is applied and results are compared with methods currently used to demonstrate how the rankings change. The method can be adapted to other areas of the shipping industry such as classification societies or ship management companies.
\end{abstract}

\section{Keywords:}

Performance measurement, small sample sizes, inspections, detentions, incidents, measuring effort under incomplete information, accounting for bad luck, incentives.

\section{Disclaimer for Ji and Knapp:}

The views expressed in this article represent those of the authors and do not necessarily represent those of the Australian Maritime Safety Authority (AMSA) and the Netherlands Bureau for Economic Policy Analysis

\footnotetext{
${ }^{1}$ CPB Netherlands Bureau for Economic Policy Analysis, PO Box 80510, 2508 GM, Den Haag, NL, tel: 070-3386000, email: X.Ji@cpb.nl;

${ }^{2}$ Econometric Institute, Erasmus University Rotterdam, PO Box 1738, 3000 DR, Rotterdam, NL, tel: 010-4081364, email: brinkhuis@ese.eur.nl

${ }^{3}$ Econometric Institute, Erasmus University Rotterdam, PO Box 1738, 3000 DR, Rotterdam, NL, email: knapp@ese.eur.nl;
} 


\section{Introduction}

The shipping industry is characterized by a complex legislative framework of over 50 conventions of the International Maritime Organization (IMO), which lacks enforcement powers due to its international nature. Since enforcement at the flag state level is not directly monitored, port states have created port state control regimes (PSC) that enforce internationally agreed standards on vessels entering their territory, by exercising their right to perform PSC inspections. If a vessel is found to be not compliant, it can be detained. Two PSC regimes (the Paris MoU and the Tokyo MoU) publish each year a list of flags according to their performance during inspections, the so-called Black/Grey/White List (BGW-list), where black listed flags perform worst. The Paris MoU covers the European Union, parts of Canada and the Russian Federation while the Tokyo MoU covers Asia, Australia, Chile and parts of the Russian Federation. The list has become the industry standard and is often interpreted as a rank of list according to flag performance.

It has been a longstanding problem to find a better method to measure the performance of registries. Perepelkin et al. [1] have proposed a method that deals with some of the shortcomings of the current method, giving a common criterion. Indeed, the criterion used at the moment is defined in terms of the excess factor, the value of which depends on the BGW-list and for each of the three, black/grey/white, it is defined by a different procedure (Perepelkin et al. [1]). Perepelkin et al. [1] have considered incident data and deficiencies besides the current standard of using detention data.

Given this situation, this article builds on some aspects of the method developed by Perepelkin et al. [1], and in particular it tries to address the lack of any common criterion that depicts the effort of a flag. The proposed method introduces the concept of and indirect measure denoted the 'enforcement effort' which cannot be directly observed. The number of undesirable events is counted that are the result of insufficient effort such as weighted numbers of detainments, very serious accidents and serious accidents. The outcome is taken as a proxy for the effort of a registry. The method can be extended in the future to include other quantities that can measure enforcement effort or implementation effort. Data from the Member States audit scheme of the International Maritime Organization might perhaps be useful to integrate in the future.

In principle, other factors might also be relevant, such as the age of the vessel and the sizes or the ship type, as these have an influence on the safety quality of ships (Bijwaard and Knapp [2]). 
The reason for this is that the major shipping markets have different characteristics. These differences are due to the varying commercial conditions of the shipping markets and are best reflected by ship types. Ship types are not considered in methods currently used to measure performance. Moreover, it is also difficult to evaluate a registry with a small fleet fairly by means of currently used methods. One reason for this is that for small fleet, the performance is more prone to bad luck. Therefore, the concept of 'sympathy' is introduced into the measure, giving each flag the benefit of the doubt, but not more. Registries with smaller fleets get more sympathy, as desired.

The proposed method also addresses the lack of use of combined data sources (Knapp [3], Knapp and Franses [4], Bijwaard and Knapp [2]) such as combined inspection data or incident data to provide a more complete picture of the enforcement effort. The use of incident data is also extended to include two degrees of seriousness - very serious and serious incidents.

The challenge is to find a method to measure enforcement effort of international standards, providing leniency to registries that have smaller fleets or that have more challenging fleet profiles. The second challenge is to provide fair incentives to improve. It is reasonable to expect that in case of sufficient effort by a flag, for the ships under this flag, certain undesirable events will be rare. For example, then inspections of ships will rarely lead to detention, and very serious incidents will be rare. This suggests to count some well-chosen types of undesirable events, detentions and very serious accidents, and to use the outcome as a performance measurement that is proxy for the effort: a low respectively high outcome is interpreted as a good respectively inadequate effort by the flag.

The method is applied and results are compared with methods developed by Perepelkin et al. [1] and with the excess factor methods currently used by the industry in order to demonstrate how the ranking of flags changes by introducing the 'enforcement effort' and 'sympathy' to registries with smaller fleet or with more challenging fleet profiles.

The proposed method is not restricted to the use of registries but could be extended to recognized organizations (RO) or Document of Compliance Companies or any other agent where the principal cannot be directly observe the effort, but only certain undesirable events that must be ascribed to a mixture of chance and inadequate effort, that is, in many moral hazard problem (see Laffont and Martimort [5] for this type of problem). 


\section{Derivation of proposed method}

\subsection{General concept}

The development of the alternative method starts with the introduction of two numbers for each flag $F, d_{F}$, the quotient of the proportion of inspections of vessels under flag $F$ that lead to detention and this proportion for all vessels, and $z_{F}$, the quotient of the proportion of the vessels under flag $F$ that has been involved in a very serious accident and this proportion for all vessels. Thus, one gets that for $d_{F}$, as well as for $z_{F}$, the value 1 is a benchmark. For example, $z_{F}$ is smaller, respectively larger, than 1 precisely if the proportion of vessels under flag $F$ that has been involved in a very serious accident is smaller, respectively larger, than this proportion for all flags. It follows that the of two flags is compared, $F_{1}$ and $F_{2}$, for which $d_{F_{1}} \geq d_{F_{2}}$ and $z_{F_{1}} \geq Z_{F_{2}}$ : in this case, one considers that the effort of $F_{2}$ is at least as good as that of $F_{1}$.

This idea is now extended in order to able to compare the effort for each pair of flags. To this end, a weight factor $c$ is introduced which is to be chosen by policy makers. As such, one can consider that the effort of $F_{2}$ is at least as good as that of $F_{1}$ precisely if $d_{F_{1}}+c Z_{F_{1}} \geq d_{F_{2}}+$ $c Z_{F_{2}}$. That is, a first attempt is made for measuring the performance of a flag $F$ :

$$
Q_{F}{ }^{\prime}=d_{F}+c z_{F} \quad \text { 'crude performance measure’ (1) }
$$

The lower this number is, the better the effort of the flag to enforce standards. This measure is a combination of inspections, detentions, very serious incidents and fleet size. Note that for a flag with an average number of detentions and very serious accidents, the performance measure is $1+c$

There are two other types of undesirable events that could be considered as well. There is the deficiency information from PSC inspections, and there is the number of accidents or incidents. Deficiency information is used in the Perepelkin et al. [1]. The proposed method does not include deficiencies since detention information covers the main aspects of the undesirable event associated with inspections. In addition, there are about 400 different types of deficiencies which have to be grouped and weighted by policy makers according to their importance which is in essence captured by detention. For this reason, the method is extended to include two types of incidents, extending the use of very serious incidents from Perepelkin et. al. [1]. This will 
provide three levels of seriousness of undesirable events - detentions, serious and very serious incidents. In theory, this could be extended with other information such as outcomes of audits from the member states audit scheme of the International Maritime Organization in the future.

\subsection{Introduction of ship types as proxy to different fleet profiles}

In order to better quantify the effort of a flag, the method distinguishes between ship types based on Knapp [3] as follows: 1) general cargo, 2) dry bulk carrier, 3) container vessel, 4) tankers, 5) passenger vessels and 6) all other ship types. Other reasons for taking ship type into account are that ship types can also be used as a proxy for other factors such as age or size (refer to Table 1). Some registries administer a fleet of older ships of high risk ship types such as general cargo vessels (Knapp [3], Knapp [6], Knapp et al.[7], ) which tend to engage in more regional trade compared to for instance large tankers, container vessels or dry bulk carriers. Monitoring a fleet of older ships engaged in local trade is more challenging than monitoring for instance a fleet of young tankers. Nevertheless, the same effort will lead on average (refer to Table 1) to a higher detention rate.

Table 1: Descriptive statistics ship types (2006 to 2008)

\begin{tabular}{|l|r|r|r|r|r|}
\hline Ship type & $\begin{array}{r}\text { Age } \\
\text { Mean }\end{array}$ & $\begin{array}{r}\text { GRT } \\
\text { Mean }\end{array}$ & $\begin{array}{r}\text { Deficiencies } \\
\text { Mean }\end{array}$ & $\begin{array}{r}\text { Detention } \\
\text { rate }\end{array}$ & $\begin{array}{r}\text { Incident rate } \\
\text { (very serious) }\end{array}$ \\
\hline General cargo & 18.7 & 9,326 & 4.08 & $7.0 \%$ & 0.0031 \\
Dry bulk carriers & 14.4 & 31,462 & 2.82 & $4.0 \%$ & 0.0021 \\
Container ships & 9.7 & 33,885 & 1.79 & $1.8 \%$ & 0.0020 \\
Tankers & 10.1 & 29,959 & 1.85 & $2.3 \%$ & 0.0009 \\
Passenger ships & 18.9 & 33,626 & 3.00 & $2.4 \%$ & 0.0020 \\
Other ship types & 20.8 & 4,315 & 3.96 & $8.1 \%$ & 0.0099 \\
\hline
\end{tabular}

The first improvement to the crude formula $\quad Q_{F}{ }^{\prime}=d_{F}+c z_{F}$ for measuring the performance of a flag $F$ is made by making a small change: that is the different ship takes are accounted for and this gives the following improved formula for measuring the performance of a flag $F$ :

$Q_{F}=\frac{1}{N_{F}} \sum_{t \in F}\left(\alpha_{t} * D_{t, F}\right)+\frac{c}{N_{\text {ships }}} \sum_{t \in F}\left(\beta_{t} * Z_{t, F}\right) \quad$ 'finer performance measure' (2)

where:

$F$ : a flag,

$Q_{F}$ : the performance measure of flag $F$;

$N_{F}$ : the number of inspections of ships under flag $F$ during the period under consideration; 
$N_{\text {ships }_{F}}$ : the number of ships under flag $F$, averaged over the period under consideration;

$c$ : a positive constant, to be chosen by policymakers, that gives the weight of a very serious casualty compared to a detention;

$t:$ a type of ship, determined by age and tonnage group;

$t \in F$ : shorthand notation for 'the types of ship that occur among the ships under flag $F$ ';

$D_{t, F}$ : the number of detentions of ships of type $t$ under flag $F$ during the period under consideration;

$Z_{t, F}$ : the number of very serious incidents of ships of type $t$ under flag $F$ during the period under consideration;

The coefficients $\alpha_{t}$ and $\beta_{t}$ in the formula above are calculated with the following formulas:

$\alpha_{t}=\frac{N_{t}}{D_{t}}$ provided $D_{t}$ is not zero, where $D_{t}$ is the number of detentions of ships of type $t$ of all flags during the period under consideration, and $N_{t}$ is the number of inspections of ships of type $t$ of all flags during the period under consideration; if $D_{t}=0$, then we put $\alpha_{t}=0$, for example (it does not matter what we put here, as in the summation $\alpha_{t}$ is multiplied with $D_{t, F}$, which is zero if $D_{t}=0$ ).

$\beta_{t}=\frac{N_{\text {ships }_{t}}}{Z_{t}}$ if $Z_{t}$ is not zero, where $N_{\text {ships }_{t}}$ is the number of ships of type $t$ of all flags during the period under consideration, and where $Z_{t}$ is the number of very serious incidents of ships of type $t$ for all flags, during the period under consideration; if $Z_{t}=0$, then we put $\beta_{t}=0$, for example (with a similar justification as given above for $\alpha_{t}$ ). Including serious incidents as well, we obtain the following formula:

$Q_{F}=\frac{1}{N_{F}} \sum_{t \epsilon F}\left(\alpha_{t} * D_{t, F}\right)+\frac{c}{N_{\text {ships }_{F}}} \sum_{t \in F}\left(\beta_{t} * Z_{t, F}\right)+\frac{d}{N_{\text {ships }_{F}}} \sum_{t \in F}\left(Y_{t} * S_{t, F}\right)$

where:

$d$ : a positive constant, to be chosen by policymakers, that gives the weight of a serious casualty compared to a detention;

$Y_{t}=\frac{N_{\text {ships }} t}{S}$ if $S_{t}$ is not zero, where $S_{t}$ is the number of serious incidents of ships of type $t$ for all flags, during the period under consideration; if $S_{t}=0$, then we put $\Upsilon_{t}=0$, for example (with a similar justification as given above for $\alpha_{t}$ ).

$S_{t, F}$ : the number of serious incidents of ships of type $t$ under flag $F$ during the period under consideration. 
The reason for the chosen correction for detentions is as follows: without corrections for ship types, one would take for the contribution of the detentions to the measure of the performance of flag $F$, the ratio $\frac{D_{F}}{N_{F}}$, where $D_{F}$ is the number of detentions of ships under flag $F$. This can be written as $\frac{1}{N_{F}} \sum_{t \in F} D_{t, F}$. To make the numbers of detentions comparable between different types,

it is reasonable to multiply, for each type $t$, the term $D_{t, F}$ by $\alpha_{t}=\frac{N_{t}}{D_{t}}$, the average number of inspections for one detention for ships of type $t$. This gives the contribution $\frac{1}{N_{F}} \sum_{t \in F}\left(\alpha_{t} * D_{t, F}\right)$ to the measure of the enforcement effort of flag $F$. In particular, this will make the contribution of the detentions of old ships smaller, as desired. The reason for the chosen correction for very serious (and serious) incidents is the same. In particular, for a flag that has an average number of detentions and very serious incidents, the enforcement effort will be $1+c$. For the variant that takes serious incidents into account, this is $1+c+d$.

\subsection{Adaptation for small fleet sizes}

The finer measure of the performance of a flag given above, $Q_{F}$, is not an adequate measure for the performance of flags with a small fleet. The numbers $D_{t, F}$ and $Z_{t, F}$ are subject to chance, they are stochasts, and for small fleet the effect of chance on the outcomes (for example, 'bad luck'), and so on the outcome of the formula, can be unacceptably large. Ideally one would like to replace in the formula the random variables by their expectations, but these are unknown. Therefore Appendix A presents a systematic method to replace the numbers $D_{t, F}$ and $Z_{t, F}$ by numbers that are slightly smaller, just enough to make sure, within a certain precision, that these numbers are smaller than the expectations of these random variables. This makes the outcome of the formula smaller, and so a flag is certain not to be given by bad luck a performance measure $Q_{F}$ that is higher than deserved; to be more precise: it is very unlikely that this will happen. That is, the qualities of the flags are attributed with some sympathy, 'the benefit of the doubt'. We will see that this systematic way accords more sympathy to flags with a small fleet than to flags with a larger fleet, as desired. Thus the shortcoming of the finer performance measure above will have been repaired. For the precise formula and the derivation and justification for it, please refer to Appendix A. 


\section{Application of proposed method and improvement incentive}

This article applies and compares three methods as follows: 1) the current excess factor method (EF) used by the Paris MoU, 2) the method proposed by Perepelkin et al. [1] and 3) the proposed new method with the incorporation of the enforcement effort and sympathy. Two variations of undesirable events are considered - that is very serious incidents only, and both incident categories. This article does not mention the name of individual flags due to the political nature of the subject matter. The main interest lies in applying the proposed method and in demonstrating how the ranks of the flags change by applying the different methods rather than producing a list to 'name and shame' of registries.

The terminology currently used such as Black/Grey/White is replaced by an alternative grouping which better describes the safety quality of vessels of companies and which in turn is reflected by the enforcement effort of a registry. It is also proposed that the values are determined every three to five years in order to give registries the opportunity to demonstrate improvement. The categories as follows:

- the worst quartile according to Q-ranking is called high risk (proxy to low effort); this corresponds according to experts reasonably well to substandard performance of a flag;

- $\quad$ the second worst quartile is called medium risk (proxy to medium effort);

- $\quad$ the best two quartiles are called low risk (proxy to high effort).

The proposed grouping goes beyond the idea of targeting substandard ships for inspection since the undesirable events include three categories with different degrees of seriousness. The method is applied to data from 2006 to 2008 of 183 thousand inspections, 8,646 detentions from the Paris MoU, the USCG, the Indian Ocean MoU and the Vina del Mar Agreement, although inspection data are not available for each of these regimes for the entire time period. The data is further complemented with incident data from Knapp [6] from four sources (IHS-Maritime, Lloyds List Intelligence Services, International Maritime Organization and the Australian Maritime Safety Authority). For the classification of seriousness, internationally agreed definitions of IMO [8] are used and very serious (524) and serious $(3,883)$ incidents are considered. The incident data needed to be manually reclassified in order to ensure compatibility of the four sources. Since fleet data was not entirely available for the entire time period by major ship types, estimates of the number of ships for each ship type and flag was used. The relevant ship types used were general cargo, dry bulk, container, tanker, passenger vessels and other ship types. The input data for the method is listed below: 
- Total number of inspections by ship type and flag

- Total number of detentions by ship type and flag

- Total number of very serious and total number of serious incidents by ship type and flag

- Total number of vessels in service by ship type for each flag

- A weight factor for $c$ for very serious incidents and a weight factor d for serious incident, relative to detention, to be determined by policy makers

In order to be able to make comparisons across the methods, registries that can be compared across all methods are chosen - that is flags with a minimum sample size of 30 inspections (based on the currently used for the EF method). From a total of 132 flags, one can evaluate 99 flags across all three methods. There are many different variations one could compare but for the sake of demonstration, the following four combinations are considered:

- Current excess factor method where only detentions are considered

- Method based on Perepelkin et al.[1] where detentions and very serious incidents are considered with weight factors $c=4$ and $c=5$ respectively

- $\quad$ The proposed new method with information by ship type for detentions and very serious incidents with weight factors $c=4$ and $c=5$ respectively

- The proposed new method with information by ship type for detentions, very serious and serious incidents with weight factors $c=4$ and $d=2$ and for $c=5$ and $d=3$ for very serious/serious incidents respectively

\section{Discussion of results}

The combinations above are applied and the flags are ranked best to worst (1 meaning best and 99 meaning worst rank or least effort). The first comparison relates to the EF method with each of the combinations and different weight factors $c$ (very serious incidents) and $d$ (serious incidents) to see how the ranks change and a series of graphs are presented in Appendix B for easy comparison. On can observe some agreement compared to the method in current use but also large changes in rank for a number of flags. The change in ranks reflects the effect of incorporating incidents (either serious or very serious) compared to detentions or deficiencies only. At least 20 flags with change of up to 50 ranks are identified.

The change of ranks of the new proposed method compared to the EF method can further be explained by the incorporation of the ship types, which accounts for the differences in the fleet 
profiles. This is because, more `sympathy’ is provided tor maritime administrations that register for instance older general cargo ships or have varying fleet profiles in general. To provide more insight into this, Table 2 provides the values for the three parameters - alpha, beta and gamma for each ship type based on the sample data used (given in equation 3 earlier).

Table 2: Weight factors for leniency by ship type

\begin{tabular}{|l|r|r|r|}
\hline Ship type & $\boldsymbol{\alpha}_{\boldsymbol{t}}$ & $\boldsymbol{\beta}_{\boldsymbol{t}}$ & \multicolumn{1}{c|}{$\boldsymbol{\gamma}_{\boldsymbol{t}}$} \\
\hline general cargo & 14.45 & 328.78 & 54.14 \\
dry bulk carriers & 24.87 & 474.47 & 48.98 \\
container ships & 56.79 & 602.54 & 42.36 \\
Tankers & 44.21 & 1052.95 & 89.80 \\
passenger ships & 44.12 & 483.46 & 40.51 \\
other ship types & 20.25 & 95.90 & 21.12 \\
\hline
\end{tabular}

The parameters are weight factors for detentions $\left(\alpha_{t}\right)$, very serious incidents $\left(\beta_{t}\right)$ and serious incidents $\left(\gamma_{t}\right)$ for a certain ship type $t$. The larger the weight factor $\alpha_{t}$, the smaller is the probability for the ship type to be detained. This also holds for serious and very serious incidents. Due to the fact that very serious incidents are rare events, values of $\beta_{t}$ are on average much larger than the other parameters. For small weight factor values, the proposed method will provide more sympathy to the registry since it will be more challenging to administer an older fleet (e.g. general cargo) trading in coastal areas compared to vessels that trade internationally on either set routes (e.g. container trade, dry bulk trades) or are inspected more often (e.g. tankers).

Interesting to observe from Appendix B is that one can find not much difference in the effect of the chosen weight factors for either very serious incidents $(c=4$ or $c=5$ ) or serious incidents $(d=2, d=3)$. Our implementation does not show many differences in the outcomes, but the fact that it uses more data suggests a greater reliability. Therefore it seems slightly preferable to implement the method taking serious accidents into account as well. One could argue that at this moment, the population of serious incidents is incomplete since reporting to the IMO is biased. This however can change in the future and with better data population, the inclusion of serious incidents into the formula will account better for the effort since more observations are available compared to very serious incidents. The proposed new method only requires one weight factor for each incident category, to be determined by the policy makers: the weight factor for a very serious incident and the weight factor for a serious incident relative to a detention. 
Perhaps larger increases for both weights compared to detention might be more appropriate and could be investigated in the future. For the purpose of this article, it is suggested that policy makers should provide these weight factors based on expert knowledge. For the remainder of this analysis, two weight factors are chosen, namely $c=4$ for very serious and $d=2$ for serious incidents. Then the ranks are compared across these two combinations with the current excess factor method and with each other.

The top-left picture of Figure 1 for instance, gives the points $\left(x_{f}, y_{f}\right)$ for all flags $f$, where $x_{f}$ is the rank according to the excess factor and , $y_{f}$ the rank according to the method of Perepelkin et al.[1] with weight factor $c=f$ (very serious incidents only). The picture below presents the same but compared to the new proposed method with weight factor $c=4$ (very serious incidents) and $d=2$ (serious incidents). The proposed method shows more variability compared to the EF method than the method of Perepelkin et al. [1], which uses only very serious incidents and which does not make any distinction between ship types. One can also notice that there is less change in the new method compared to Perepelkin et al. [1].

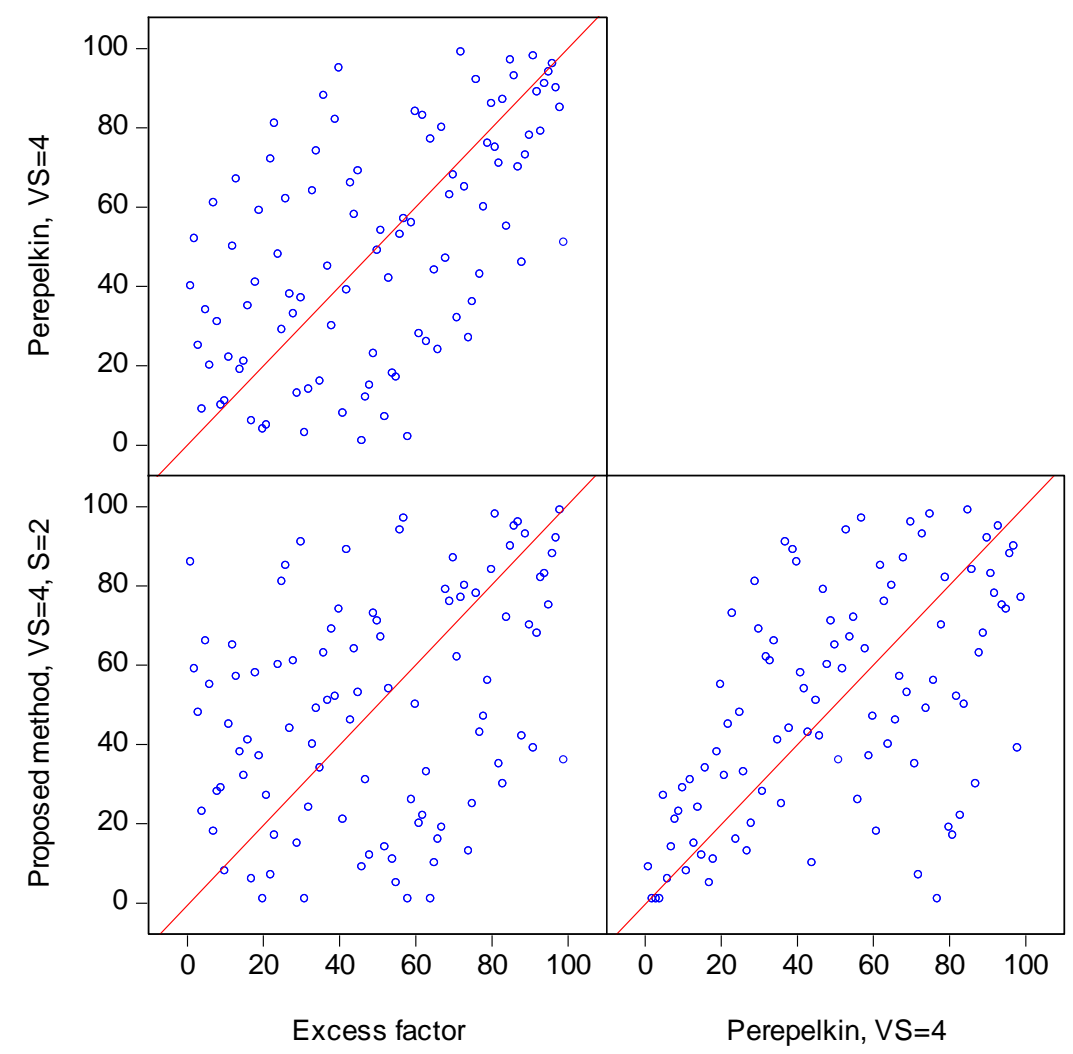

Figure 1: Comparison of ranks with various methods, VS=very serious, $S=$ =serious incidents 
The next topic of interest is to see how the risk categories change and the flags are classified according to their risk levels as mentioned earlier, where the worst quartile of $\mathrm{Q}$ is classified as high risk (proxy to low effort), the second worst quartile is medium risk (proxy to medium effort) and the remaining two quartiles are low risk flags (proxy to high effort). Appendix C provides selected results for the different combinations and weight factors with the changes of the risk categories for all flags that were evaluated. Not surprisingly by now, given Figure 1, one can observe many shifts from the high risk category based on the EF method to the medium or low risk category based on either Perepelkin et al. [1] of the new proposed method. When including serious incidents, one can further observe shifts of some flags from the low risk category to the medium risk category and vice versa, compared to the method by Perepelkin et al.[1].

Some of the shifts from high risk or medium to low risk can be explained by a combination of ship types - so maritime administrations with general cargo vessels were given more sympathy. Shifts from the low risk category to the medium risk category can be explained by very serious incidents on tankers and passenger vessels, and therefore receive less sympathy since in general it will take less effort to administer these ship types. Other shifts are simply due to the addition of serious incidents. Nine flags do not change across the methods and remain in the 'high risk' category irrespective of the method applied. These flags perform consistently worse even though their fleet profile is strongly characterized by a high proportion of general cargo vessels or in one case a combination of general cargo vessels and tankers. However, even if sympathy is given, these flags show the least effort in enforcing international standards.

\section{Conclusions and recommendations}

This article proposes a more refined method to measure the effort of a flag to administer its fleet given that some registries have varying fleet profiles or take vessels into their registry that might be more challenging to administer (e.g. older general cargo vessels, smaller fleet profiles, etc.). The method is applied and results are compared against two other methods, in particular how the ranks of some flags change. The advantages of the new method over the other methods can be summarized as follows. The new method is based on one extremely simple and convincing idea: counting undesirable events and using this as a proxy for the effort of a flag, which cannot be measured directly. There is no need for policy makers to determine the many weight factors for various types of deficiencies as only two weight factors are needed. In this way, policy makers 
have the possibility to fine-tune the method by choosing one (or two) weight factors. More information is used compared to the method currently used and so the sample bias is smaller and more accurately reflects reality.

Distinction of ship types can be taken into account, and this is strongly desirable because of the impact of different market characteristics on safety. Some sympathy is given to registries with more challenging fleet profiles and/or small fleet; the latter are more susceptible to bad luck. For more challenging fleet types, this is done by distinguishing ship types. For small fleet, some sympathy is given to each flag to account for possible bad luck and this is done in a systematic way such that the sympathy given is just enough to account for possible bad luck of flags. This sympathy is greater for small flags, as is desired: these flags are more susceptible to bad luck. Although not demonstrated here, the proposed method can also evaluate flags with smaller sample sizes (below 30).

The proposed method of counting undesirable events is very flexible and easy to implement practically. The flexibility is demonstrated by giving straightforward adaptations from the basic counting idea that take into account all issues that arise in practice and that lead to the shortcomings of the current excess factor method, partly addressed by the method developed by Perepelkin et al. [1].

Based on this analysis, it is recommended that policy makers use the following undesirable events as proxy to determine the effort in enforcing international standards: detentions, serious accidents and very serious incidents. With respect to the use of serious incidents, the method will become more precise once better data is being populated by the IMO via the Global Integrated Ship Information System (GISIS). We also feel that a change in terminology from the current division of Black/Grey/White into High/Medium/Low Risk, where the first two groups are the worst two quartiles, is more appropriate. In addition, the method could be extended to include other information such as data from the member state audit schemes of the International Maritime Organization.

Another recommendation is to fix the boundaries between these three groups for three years, based on data from the last three years (possibly use five years' worth of data in the future), and to give flags the opportunity to move upward, especially from High Risk to Medium Risk, in order to reduce occurrence of substandard ships and improve overall safety. Finally, the method 
should be implemented in such a way that no name-and-shame effects can arise for flags from changes in ranks compared to the method currently in use.

\section{Acknowledgements}

The authors would like to thank our data providers for the provision of incident and fleet data, in particular IHS-Maritime, LLIS and the International Maritime Organization.

\section{References}

[1] Perepelkin M, Knapp S, Perepelkin G and de Pooter M (2010), A method to measure flag performance for the shipping industry, Marine Policy, Volume 34(3), pages 395-40

[2] Bijwaard G and Knapp S (2009), Analysis of Ship Life Cycles - The Impact of Economic Cycles and Ship Inspections, Marine Policy, volume 33, pp. 350-369

[3] Knapp, S (2006), The Econometrics of Maritime Safety - Recommendations to enhance safety at sea, Doctoral Thesis, Erasmus University, Rotterdam

[4] Knapp S, Franses PH (2007) A global view on port state control - econometric analysis of the differences across port state control regimes, Maritime Policy and Management, 34(5), pages 453-483

[5] Laffont J-J, Martimort D (2002), The Theory of Incentives - The Principal-Agent Model, (Princeton University Press Princeton and Oxford)

[6] Knapp S (2013), An integrated risk estimation methodology: Ship specific incident type risk, EI report 2013-11, http://repub.eur.nl/res/pub/39596/

[7] Knapp S, Heij C, Henderson R, Kleverlaan E (2013), Ship incident risk in the areas of Tubbataha and Banc d'Arguin: A case for designation as Particular Sensitive Sea Area?

[8] International Maritime Organization (2000), MSC/Circ. 953, MEPC/Circ. 372, Reports on Marine Casualties and Incidents, Revised harmonized reporting procedures, adopted $14^{\text {th }}$ December 2000, London 


\section{Appendix A: Derivation of the formula for sympathy for bad luck}

This technical section provides an analysis for the variation in the observations with the following additional notation. Let $N_{t, F}$ be the number of inspections of ships of type $t$ under flag $F$ during the period under consideration $\left(\sum_{t \in F}\left(N_{t, F}\right)=N_{F}\right)$, and let $N_{\text {ships }}$ t,F be the total number of ships of type $t$ under flag $F$, averaged over the period under consideration $\left(\sum_{t \in F}\left(N_{\text {ships }_{t, F}}\right)=\right.$ $\left.N_{\text {ships }}\right)$. Assume that the number of detentions of a certain ship type under a certain flag follows a binomial distribution: $D_{t, F} \sim \operatorname{Bin}\left(N_{t, F}, p_{t, F}^{d}\right)$, with $p_{t, F}^{d}$ the underlying probability of detention at one inspection of ship type $t$ under the flag $F$. Moreover, it is assumed that the number of very serious incidents of ship type $t$ under flag $F$ also follows a binomial distribution $Z_{t, F} \sim \operatorname{Bin}\left(N_{\text {ships }_{t, F}} p^{z}{ }_{t, F}\right)$, with $p_{t, F}^{z}$ the underlying probability of a very serious casualty for each ship of type $t$ under flag $F$. The probabilities of detention or very serious casualties of one ship type differ among the flags because of the differences in management among the flags, the very effect which is the intention to measure. When $N_{t, F}$ is large enough, the distribution of the stochast $D_{t, F}$ approximates the normal distribution with

$E\left(D_{t, F}\right)=N_{t, F} * p_{t, F}^{d}$ and $\operatorname{Var}\left(D_{t, F}\right)=N_{t, F} * p_{t, F}^{d} *\left(1-p_{t, F}^{d}\right)$.

The same holds for the distribution of the random variable $Z_{t, F}$ : when $N_{\text {ships }}$ is is large enough, the distribution of the random variable $Z_{t, F}$ approximates a normal distribution, with $E\left(Z_{t, F}\right)=$ $N_{\text {ships }_{t, F}} * p_{t, F}^{z}$ and with $\operatorname{Var}\left(Z_{t, F}\right)=N_{\text {ships }_{t, F}} * p_{t, F}^{z} *\left(1-p_{t, F}^{z}\right)$. It will be convenient to write $\left(p_{t, F}^{d}\right)^{\prime}=\frac{D_{t, F}}{N_{t, F}}$ and to view this as an observation of a normally distributed random variable with mean $p_{t, F}^{d}$ and variation $\frac{p_{t, F}^{d}\left(1-p_{t, F}^{d}\right)}{N_{t, F}}$. Similarly for $\left(p_{t, F}^{z}\right)^{\prime}=\frac{z_{t, F}}{N_{\text {ships }_{t, F}} \text {. }}$

Ideally one would like to take as a measure for the performance of a flag:

$$
Q_{F}^{*}=\frac{1}{N_{F}} \sum_{t \epsilon F}\left(\alpha_{t} * N_{t, F} * p_{t, F}^{d}\right)+\frac{C}{N_{\text {ships } F}} \sum_{t \epsilon F}\left(\beta_{t} * N_{\text {ships }_{t, F}} * p_{t, F}^{z}\right)
$$


This formula has been obtained from the one for $Q_{F}$ by replacing $D_{t, F}=N_{t, F} *\left(p_{t, F}^{d}\right)^{\prime}$ by $N_{t, F} *$ $p_{t, F}^{d}$, and by replacing $Z_{t, F}=N_{\text {ships }_{t, F}} *\left(p_{t, F}^{z}\right)^{\prime}$ by $N_{\text {ships }}{ }_{t, F} p_{t, F}^{z}$. Unfortunately, one cannot observe the underlying probabilities.

Therefore, systematic ‘lower bounds’ for these probabilities are taken (refer to Perepelkin et al. [1]) using a systematic way to derive these 'lower bounds'. Let $p^{\prime}$ be the observed value of the random variable. Thus, $p$ stands for $p_{t, F}^{d}$ respectively $p_{t, F}^{z}$, and $p^{\prime}$ stands for $\frac{D_{t, F}}{N_{t, F}}$ respectively $\frac{Z_{t, F}}{N_{\text {ships }_{t, F}}}$. The standard deviation is $\sigma=\sqrt{\frac{p(1-p)}{N}}$. A confidence interval is fixed for a confidence level $a$ (for example, $a=0.95$ is a popular choice) and the confidence interval ( $p-$ $c_{a}, p+c_{a}$ ) is defined for each $p$ such that a value $p^{\prime}$ of the stochast is contained in this interval with probability $a: \operatorname{Pr}\left[\left|p^{\prime}-p\right|<c_{a}\right]=a$.

The meaning of this interval is as follows. The values of $p$ that satisfy this condition are the values of $p$ for which the hypothesis that $p^{\prime}$ lies in the confidence interval of the normal distribution with mean $p$ and standard deviation $\sigma$ cannot be rejected with confidence level $a$. To put it more simply, but not entirely precisely: one can be 'sure' that the true value of the underlying probability $p$ does not differ more than $c_{a}$ from the observed value $p^{\prime}$ (here 'sure' means roughly that the probability that this statement is incorrect for given $p$ equals $1-a$ ). The method takes $L(p)$, the smallest value of $p$ that satisfies this condition as the 'lower bound' of $p$. The reason for this terminology is that for given $p$, one can be confident that $L(p)$ is smaller than $p$, given the prescribed confidence level $a$. Because of the fact that $p^{\prime}$ is normally distributed, it holds that: $\operatorname{Pr}\left[\left|p^{\prime}-p\right|<c_{a}\right]=2 * \Phi_{\frac{c_{a}}{\sigma}}-1$, where $\Phi$ is the cumulative function of the standard normal distribution and $\Phi_{\frac{c_{a}}{\sigma}}$ gives the cumulative probability for $Z \leq \frac{c_{a}}{\sigma}$ and $Z \sim N \operatorname{Norm}(0,1)$. It follows that: $\left|p^{\prime}-p\right|<\Phi^{-1}\left(\frac{1+a}{2}\right) \sigma$ and from the above, it follows:

$$
\begin{aligned}
& \left|p^{\prime}-p\right|<t_{a} \sqrt{\frac{p(1-p)}{N}} \text { with } t_{a}=\Phi^{-1}\left(\frac{1+a}{2}\right) \\
& \left(p^{\prime}-p\right)^{2}<t_{a}^{2} * \frac{p(1-p)}{N} \\
& p^{\prime 2}-2 p^{\prime} p+p^{2}<\frac{t_{a}^{2}}{N} p-\frac{t_{a}^{2}}{N} p^{2} \\
& \left(1+\frac{t_{a}^{2}}{N}\right) p^{2}+\left(-2 p^{\prime}-\frac{t_{a}^{2}}{N}\right) p+p^{\prime 2}<0 .
\end{aligned}
$$


This is an inequality in a quadratic polynomial in $p$. As $1+\frac{t_{a}{ }^{2}}{N}$, the coefficient of $p^{2}$, is positive, the solutions form an open interval with endpoints the roots of the quadratic equation $\left(1+\frac{t_{a}^{2}}{N}\right) p^{2}+\left(-2 p^{\prime}-\frac{t_{a}^{2}}{N}\right) p+p^{\prime 2}=0$.

Then, the abc-formule can be used to get the 'lower bound' for $p$ :

$L(p)=\frac{-\left(-2 p^{\prime}-\frac{t_{a}{ }^{2}}{N}\right)-\sqrt{\left(2 p^{\prime}+\frac{t_{a}{ }^{2}}{N}\right)^{2}-4\left(1+\frac{t_{a}{ }^{2}}{N}\right)\left(p^{\prime 2}\right)}}{2\left(1+\frac{t_{a}{ }^{2}}{N}\right)}$

The other root is the 'upper bound', denoted by $U(p)$. For flags with small fleet, the variation in the random variable $p^{\prime}$ is larger. Indeed, a good measure for this variation is the difference $U(p)-L(p)$. As the denominator in the expression $L(p)$ (and $U(p)$ ) is approximately 2, one can see that $(U(p)-L(p))^{2}$ is approximately $\left(2 p^{\prime}+\frac{t_{a}^{2}}{N}\right)^{2}-4\left(1+\frac{t_{a}^{2}}{N}\right)\left(p^{\prime 2}\right)$. This can be rewritten as $\frac{t_{a}^{4}}{N^{2}}+\frac{2 p^{\prime} t_{a}}{N}\left(t_{a}-p^{\prime}\right)$. As $t_{a} \approx 2$ (usually) and $p^{\prime} \in(0,1)$, one can see that this is decreasing in $N$. Thus, for flags with small fleet, the variation is large.

The above is applied to the probabilities of detention and of very serious incidents. After simplification, the promised 'lower bounds' of probabilities of detention and very serious incidents for different ship type and different flags is obtained:

$L\left(p_{t, F}^{d}\right)=\frac{\frac{D_{t, F}}{N_{t, F}}+\frac{1}{2} * \frac{t_{a}{ }^{2}}{N_{t, F}}-t_{a} \sqrt{\left(\frac{D_{t, F}\left(N_{t, F}-D_{t, F}\right)}{N_{t, F}{ }^{3}}\right)-\frac{1}{4} * \frac{t_{a}{ }^{2}}{N_{t, F}{ }^{2}}}}{1+\frac{t_{a}{ }^{2}}{N_{t, F}}}$

and

$$
L\left(p_{t, F}^{z}\right)=\frac{\frac{Z_{t, F}}{N_{\text {ships }_{t, F}}}+\frac{1}{2} * \frac{t_{a}{ }^{2}}{N_{\text {ships }_{t, F}}}-t_{a} \sqrt{\left(\frac{Z_{t, F}\left(N_{\text {ships }_{t, F}}-Z_{t, F}\right)}{N_{\text {ships }_{t, F}}{ }^{3}}\right)-\frac{1}{4} * \frac{t_{a}{ }^{2}}{N_{\text {ships }_{t, F}{ }^{2}}}} .}{1+\frac{t_{a}{ }^{2}}{N_{\text {ships }_{t, F}}}} .
$$

Now the correct measure for the performance measure of a flag can be obtained by replacing the observed value of each random variable $\left(p_{t, F}^{d}\right)^{\prime}$ (and $\left(p_{t, F}^{z}\right)^{\prime}$ ) by the 'lower bound' $L\left(p_{t, F}^{d}\right)$ (and $L\left(p_{t, F}^{z}\right)$ ) for the mean value of the stochast, as defined above. 
$L\left(Q_{F}\right)=\frac{1}{N_{F}} \sum_{t \in F}\left(\alpha_{t} * N_{t, F} * L\left(p_{t, F}^{d}\right)\right)+\frac{c}{N_{\text {ships }_{F}}} \sum_{t \in F}\left(\beta_{t} * N_{\text {ships }}{ }_{t, F} * L\left(p_{t, F}^{z}\right)\right)$

The 'lower bound' for $Q_{F}$ is taken as the final performance measure for a flag $F$. Both shortcomings of the crude performance measure $Q_{F}{ }^{\prime}=d_{F}+c Z_{F}$ have been overcome since this measure takes into account ship types and it takes into account the variations in the observations. 


\section{Appendix B: Change of ranks for each method and weight factor}

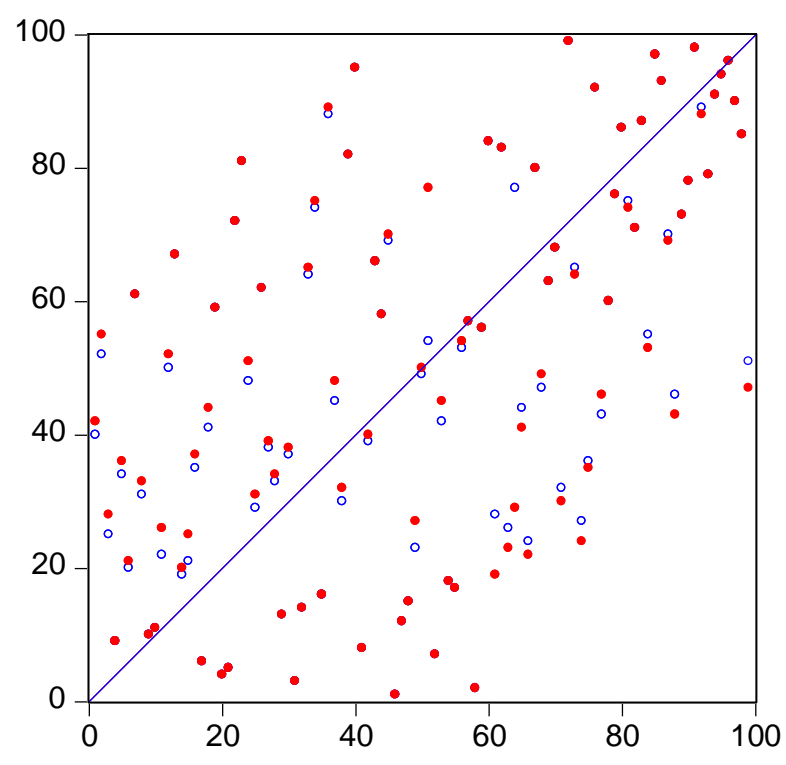

Excess factor

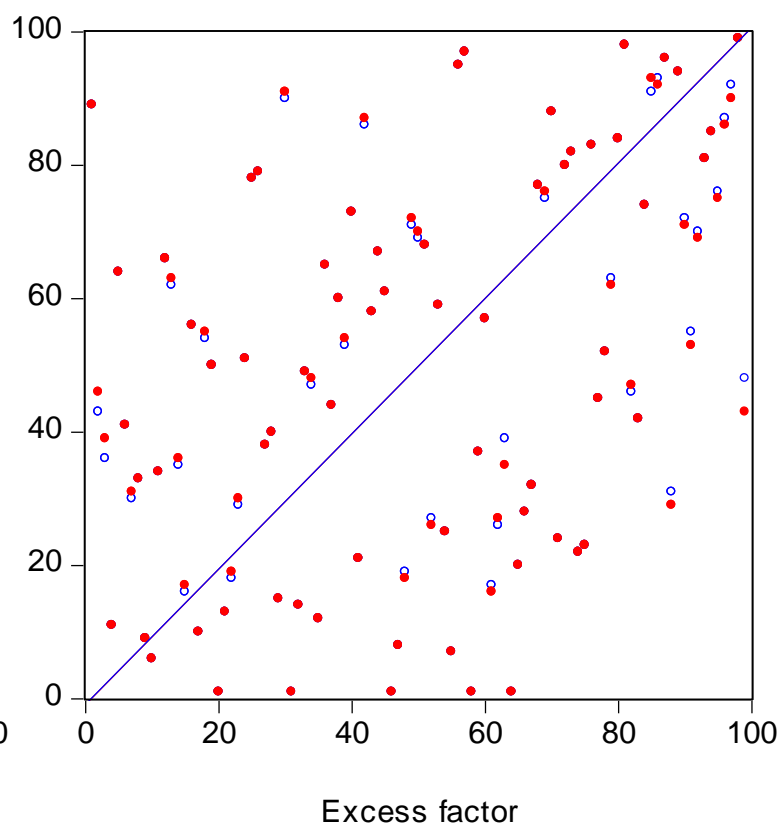

Perepelkin, very serious $=4$

- Perepelkin, very serious $=5$

- Proposed method, very serious $=4$

- Proposed method, very serious=5

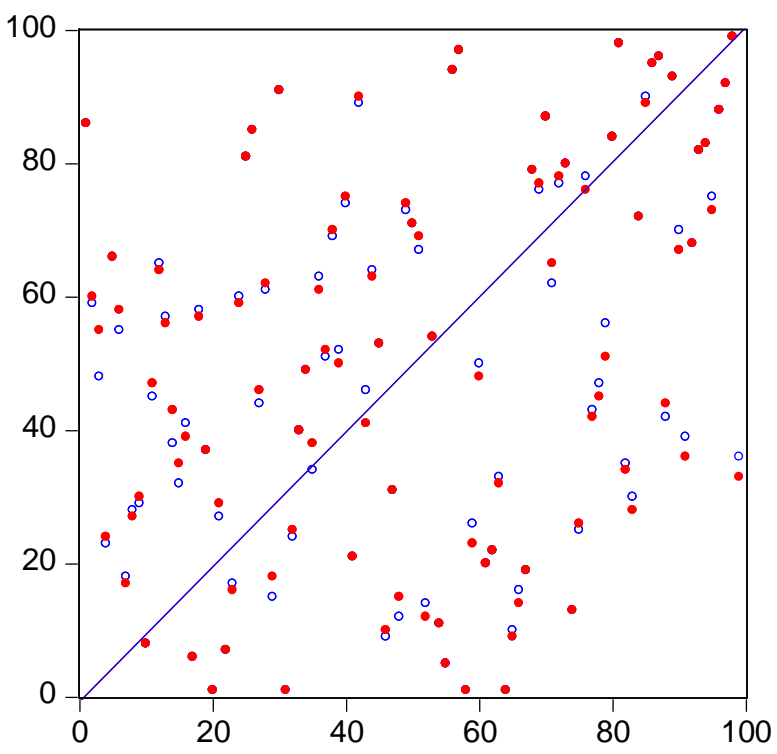

Excess factor

Proposed method, very serious $=4$, serious $=2$

- Proposed method, very serious $=5$, serious $=3$ 


\begin{tabular}{|c|c|c|c|c|}
\hline Flag & $\begin{array}{l}\text { Current method } \\
\text { excess factor } \\
\text { no weights }\end{array}$ & $\begin{array}{l}\text { Perepelkin } \\
\text { vs, } c=4\end{array}$ & $\begin{array}{l}\text { New method } \\
\text { vs, } c=4\end{array}$ & $\begin{array}{l}\text { New method } \\
\text { vs, } c=4 \\
s, d=2\end{array}$ \\
\hline Flag 1 & medium risk & low risk & low risk & low risk \\
\hline Flag 2 & low risk & low risk & low risk & low risk \\
\hline Flag 3 & low risk & low risk & low risk & low risk \\
\hline Flag 4 & medium risk & high risk & low risk & low risk \\
\hline Flag 5 & medium risk & low risk & low risk & low risk \\
\hline Flag 6 & low risk & low risk & low risk & low risk \\
\hline Flag 7 & low risk & medium risk & low risk & low risk \\
\hline Flag 8 & low risk & low risk & low risk & low risk \\
\hline Flag 9 & low risk & low risk & low risk & low risk \\
\hline Flag 10 & medium risk & low risk & low risk & low risk \\
\hline Flag 11 & medium risk & low risk & low risk & low risk \\
\hline Flag 12 & low risk & low risk & low risk & low risk \\
\hline Flag 13 & medium risk & low risk & low risk & low risk \\
\hline Flag 14 & medium risk & low risk & low risk & low risk \\
\hline Flag 15 & low risk & low risk & low risk & low risk \\
\hline Flag 16 & medium risk & low risk & low risk & low risk \\
\hline Flag 17 & low risk & high risk & low risk & low risk \\
\hline Flag 18 & low risk & medium risk & low risk & low risk \\
\hline Flag 19 & medium risk & high risk & low risk & low risk \\
\hline Flag 20 & medium risk & low risk & low risk & low risk \\
\hline Flag 21 & low risk & low risk & low risk & low risk \\
\hline Flag 22 & medium risk & high risk & low risk & low risk \\
\hline Flag 23 & low risk & low risk & low risk & low risk \\
\hline Flag 24 & low risk & low risk & low risk & low risk \\
\hline Flag 25 & high risk & low risk & low risk & low risk \\
\hline Flag 26 & medium risk & medium risk & low risk & low risk \\
\hline Flag 27 & low risk & low risk & low risk & low risk \\
\hline Flag 28 & low risk & low risk & low risk & low risk \\
\hline Flag 29 & low risk & low risk & low risk & low risk \\
\hline Flag 30 & high risk & high risk & low risk & low risk \\
\hline Flag 31 & low risk & low risk & low risk & low risk \\
\hline Flag 32 & low risk & low risk & low risk & low risk \\
\hline Flag 33 & medium risk & low risk & low risk & low risk \\
\hline Flag 34 & low risk & low risk & low risk & low risk \\
\hline Flag 35 & high risk & medium risk & low risk & low risk \\
\hline Flag 36 & high risk & medium risk & low risk & low risk \\
\hline Flag 37 & low risk & medium risk & low risk & low risk \\
\hline Flag 38 & low risk & low risk & low risk & low risk \\
\hline Flag 39 & high risk & high risk & medium risk & low risk \\
\hline Flag 40 & low risk & medium risk & low risk & low risk \\
\hline Flag 41 & low risk & low risk & medium risk & low risk \\
\hline Flag 42 & high risk & low risk & low risk & low risk \\
\hline Flag 43 & high risk & low risk & low risk & low risk \\
\hline Flag 44 & low risk & low risk & low risk & low risk \\
\hline Flag 45 & low risk & low risk & low risk & low risk \\
\hline Flag 46 & low risk & medium risk & medium risk & low risk \\
\hline Flag 47 & high risk & medium risk & medium risk & low risk \\
\hline Flag 48 & low risk & low risk & low risk & low risk \\
\hline Flag 49 & low risk & medium risk & low risk & low risk \\
\hline Flag 50 & medium risk & high risk & medium risk & low risk \\
\hline
\end{tabular}




\begin{tabular}{|c|c|c|c|c|}
\hline Flag & $\begin{array}{l}\text { Current method } \\
\text { excess factor } \\
\text { no weights }\end{array}$ & $\begin{array}{l}\text { Perepelkin } \\
\text { vs, c=4 }\end{array}$ & $\begin{array}{l}\text { New method } \\
\text { vs, } c=4\end{array}$ & $\begin{array}{l}\text { New method } \\
\text { vs, } c=4 \\
\text { s, } d=2\end{array}$ \\
\hline Flag 51 & low risk & low risk & low risk & medium risk \\
\hline Flag 52 & low risk & high risk & medium risk & medium risk \\
\hline Flag 53 & low risk & medium risk & medium risk & medium risk \\
\hline Flag 54 & medium risk & low risk & medium risk & medium risk \\
\hline Flag 55 & low risk & low risk & low risk & medium risk \\
\hline Flag 56 & high risk & high risk & medium risk & medium risk \\
\hline Flag 57 & low risk & medium risk & medium risk & medium risk \\
\hline Flag 58 & low risk & low risk & medium risk & medium risk \\
\hline Flag 59 & low risk & medium risk & low risk & medium risk \\
\hline Flag 60 & low risk & low risk & medium risk & medium risk \\
\hline Flag 61 & low risk & low risk & low risk & medium risk \\
\hline Flag 62 & medium risk & low risk & low risk & medium risk \\
\hline Flag 63 & low risk & high risk & medium risk & medium risk \\
\hline Flag 64 & low risk & medium risk & medium risk & medium risk \\
\hline Flag 65 & low risk & low risk & medium risk & medium risk \\
\hline Flag 66 & low risk & low risk & medium risk & medium risk \\
\hline Flag 67 & medium risk & medium risk & medium risk & medium risk \\
\hline Flag 68 & high risk & high risk & medium risk & medium risk \\
\hline Flag 69 & low risk & low risk & medium risk & medium risk \\
\hline Flag 70 & high risk & high risk & medium risk & medium risk \\
\hline Flag 71 & low risk & low risk & medium risk & medium risk \\
\hline Flag 72 & high risk & medium risk & medium risk & medium risk \\
\hline Flag 73 & low risk & low risk & medium risk & medium risk \\
\hline Flag 74 & low risk & high risk & medium risk & medium risk \\
\hline Flag 75 & high risk & high risk & high risk & high risk \\
\hline Flag 76 & medium risk & medium risk & high risk & high risk \\
\hline Flag 77 & medium risk & high risk & high risk & high risk \\
\hline Flag 78 & high risk & high risk & high risk & high risk \\
\hline Flag 79 & medium risk & low risk & high risk & high risk \\
\hline Flag 80 & medium risk & medium risk & high risk & high risk \\
\hline Flag 81 & low risk & low risk & high risk & high risk \\
\hline Flag 82 & high risk & high risk & high risk & high risk \\
\hline Flag 83 & high risk & high risk & high risk & high risk \\
\hline Flag 84 & high risk & high risk & high risk & high risk \\
\hline Flag 85 & low risk & medium risk & high risk & high risk \\
\hline Flag 86 & low risk & low risk & high risk & high risk \\
\hline Flag 87 & medium risk & medium risk & high risk & high risk \\
\hline Flag 88 & high risk & high risk & high risk & high risk \\
\hline Flag 89 & low risk & low risk & high risk & high risk \\
\hline Flag 90 & high risk & high risk & high risk & high risk \\
\hline Flag 91 & low risk & low risk & high risk & high risk \\
\hline Flag 92 & high risk & high risk & high risk & high risk \\
\hline Flag 93 & high risk & medium risk & high risk & high risk \\
\hline Flag 94 & medium risk & medium risk & high risk & high risk \\
\hline Flag 95 & high risk & high risk & high risk & high risk \\
\hline Flag 96 & high risk & medium risk & high risk & high risk \\
\hline Flag 97 & medium risk & medium risk & high risk & high risk \\
\hline Flag 98 & high risk & high risk & high risk & high risk \\
\hline Flag 99 & high risk & high risk & high risk & high risk \\
\hline
\end{tabular}

Note: vs=very serious, $s=$ serious 